\title{
The anxious amygdala: CREB signaling and predisposition to anxiety and alcoholism
}

\author{
Gary Wand \\ Department of Medicine and Psychiatry, Johns Hopkins University School of Medicine, Baltimore, Maryland, USA.
}

\begin{abstract}
The amygdala is believed to play a key role in assigning emotional significance to specific sensory input, and conditions such as anxiety, autism, stress, and phobias are thought to be linked to its abnormal function. Growing evidence has also implicated the amygdala in mediation of the stress-dampening properties of alcohol. In this issue of the JCI, Pandey and colleagues identify a central amygdaloid signaling pathway involved in anxiety-like and alcohol-drinking behaviors in rats (see the related article beginning on page 2762). They report that decreased phosphorylation of cAMP responsive element-binding protein (CREB) resulted in decreased neuropeptide Y (NPY) expression in the central amygdala of alcohol-preferring rats, causing high anxiety-like behavior. Alcohol intake by these animals was shown to increase PKA-dependent CREB phosphorylation and thereby NPY expression, subsequently ameliorating anxiety-like behavior. These provocative data suggest that a CREBdependent neuromechanism underlies high anxiety-like and excessive alcohol-drinking behavior.
\end{abstract}

\section{Alcohol-abuse disorders}

Alcohol-use disorders have been placed into 4 diagnostic categories of increasing severity: risky use, problem drinking, alcohol abuse, and alcohol dependence (1). This last and most severe form of alcohol disorders is characterized by loss of control over alcohol intake, tolerance, and physical dependence. It is estimated that approximately $14 \%$ of men and $5 \%$ of women in the US will experience the symptoms of alcohol abuse or dependence over their lifetimes (2). The economic cost of alcohol-use disorders is approximately $\$ 185$ billion per year (3). Many people try alcohol, but few develop alcohol dependence. This selective vulnerability is, in part, because alcohol dependence has strong genetic determinants as evidenced by family, twin, and adoption studies (4). The genetic determinants for alcoholism create a vulnerable neural substrate that interacts with alcohol to create abuse potential.

Nonstandard abbreviations used: CeA, central amygdala; CREB, cAMP responsive element-binding protein; $\mathrm{CRF}$, corticotropin-releasing factor; DA, dopamine; NP, alcohol-nonpreferring; NPY, neuropeptide Y; P, alcoholpreferring; PKA-C $\alpha, \alpha$-catalytic subunit of PKA.

Conflict of interest: The author is a consultant for Genentech, Lilly, and Solvay.

Citation for this article: J. Clin. Invest. 115:2697-2699 (2005). doi:10.1172/JCI26436.
Alcohol and the nucleus accumbens

Alcohol and alcoholism have both positive and negative reinforcing properties. The positive reinforcing properties of alcohol are linked to the hedonic aspects of alcohol intoxication. Considerable evidence shows that these positive reinforcing effects act through signal transduction systems affecting mesocorticolimbic dopamine (DA) pathways. The nucleus accumbens, a region at the base of the striatum, appears to be the key zone assigning importance to the alcohol exposure experience. Psychostimulants, opioids, and alcohol all increase synaptic DA accumulation within this important brain region (5). With PET imaging, we and others (6-8) have shown that mesolimbic DA release following drug administration is correlated with positive subjective effects. Preclinical studies have shown that drug reward can be attenuated by pharmacological or genetic manipulations that alter mesolimbic DA neurotransmission (9).

\section{Alcohol and the amygdala}

Alcohol-use disorders are also associated with negative reinforcing states. For example, individuals may drink alcohol to reduce anxiety or symptoms related to alcohol withdrawal. A large international study found strong associations between alcohol dependence and anxiety (10). Of particular interest, anxiety disorders were highly likely to predate the onset of alcoholism, lending support for a causal relationship. These data support the self-medicating hypothesis for alcohol-use disorders. This theory posits that a subset of individuals self-medicate with alcohol to reduce anxiety and/or stress (11). Additionally, an anxiety-like emotional state is often present during alcohol withdrawal and is thought to contribute to alcohol relapse in alcoholics (12).

In this issue of the JCI, Pandey and colleagues suggest that the neural substrate underlying the stress-dampening properties of alcohol is not the nucleus accumbens, but is probably the extended amygdala (13). The extended amygdala plays a crucial role not only in anxiety behaviors, but also in promoting alcohol intake (14). The amygdala receives limbic and olfactory afferents and projects fibers that innervate the hypothalamus and midbrain. Thus, the extended amygdala links the basal forebrain to the mesolimbic reward systems. This circuitry may mediate the anxiolytic effects of ethanol (14).

\section{Selectively bred rodent lines}

Rodent lines selectively bred to have a preference for alcohol over water have been an invaluable resource in the field of alcohol studies. Particularly useful lines are the alcohol-preferring (P) and alcohol-nonpreferring (NP) rats derived from a heterogeneous stock of Wistar rats at Indiana University (15). These rats are products of inbreeding highalcohol drinking and low-alcohol drinking rats, respectively. The $\mathrm{P}$ rats generally drink greater than 5 grams of alcohol per kilogram body weight per day, whereas the NP line drinks less that $1.5 \mathrm{~g} / \mathrm{kg} / \mathrm{d}$. In this issue of the JCI, Pandey and coworkers used these rats to investigate the role of $\mathrm{CAMP} / \mathrm{PKA}$ signaling in the central amygdala (CeA) as it relates to alcohol consumption and anxiety (13). The CeA is a major component of the extended amygdala. It has been identified as a site of action for negative reinforcement associated with drug abstinence (14).

The gene transcription factor cAMP responsive element-binding protein (CREB) - a component of many signaling cascades 


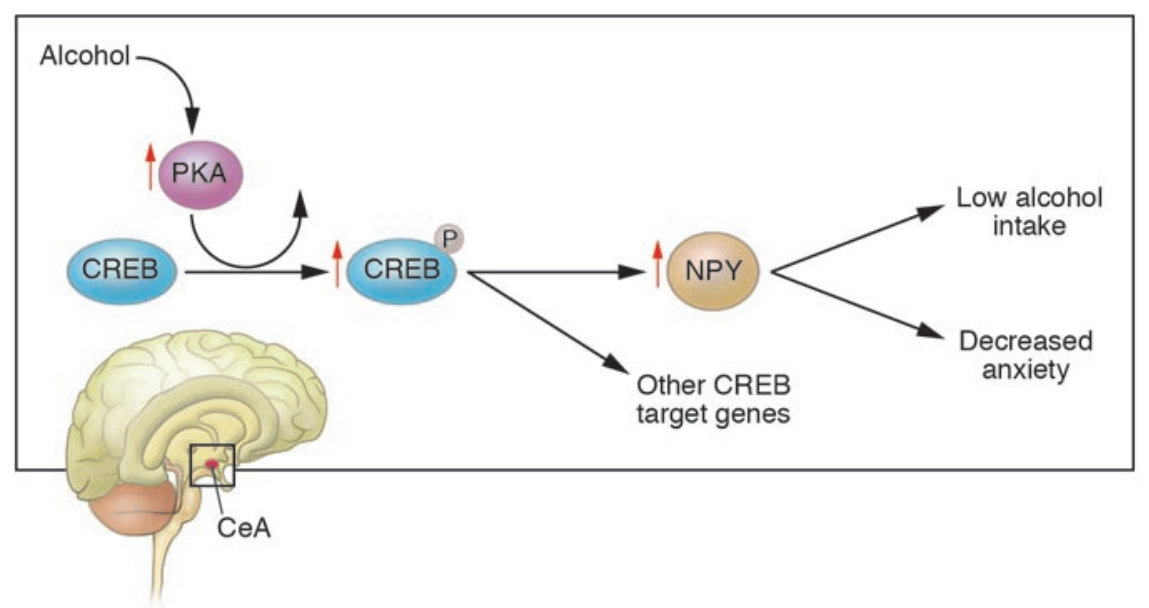

Figure 1

The amygdala and cAMP-dependent signaling. Located in the temporal lobe and forming part of the limbic system, the amygdala processes and then assigns emotional importance to specific sensory input. The amygdala has also been implicated in the generation of anxiety-like behaviors as well as mediation of the stress-dampening affects of alcohol. In this issue of the $\mathrm{JCl}$, Pandey et al. (13) show that alcohol intake activated PKA signaling, resulting in increased phosphorylation of CREB and increased NPY expression in the CeA of $P$ rats. These neurochemical changes were accompanied by decreased anxiety-like behavior. Not only were these observations mimicked by using a cAMP analog to activate PKA signaling, but alcohol self-administration in these rats was also reduced. To ensure proof of principle, the investigators infused a PKA inhibitor into the CeA of NP rats. In contrast to the results observed in P rats, the infusion provoked anxiety-like behaviors and increased alcohol intake. It appears that a CREB-dependent neuromechanism within the CeA may modulate anxiety-like behavior and alcohol intake. The findings provide a genetic model to understand how a neural substrate creates anxiety, which results in alcohol consumption to reverse a state of negative affect. The observations also open up new avenues in which to pursue pharmacological manipulation of CREB and/or NPY expression in order to modify heavy alcohol intake.

activated by neurotransmitter receptor engagement - is regulated by cAMP-dependent PKA. PKA-mediated phosphorylation of CREB regulates the downstream expression of cAMP-inducible genes including NPY (16). Many studies have shown that a relationship exists between this $\mathrm{G}$ proteinmediated signaling cascade and responses to alcohol (17). Our group initially showed that alcohol can modulate the phosphorylation of CREB (18), and several studies subsequently demonstrated that CREB plays a role in alcohol dependence and preference (19-21). For example, CREB haplodeficient mice have a higher preference for alcohol than wild-type mice (21). NPY has been shown to play a role in anxiety and alcohol abuse (22). NPY-null mice display more anxiety-like behaviors and consume more alcohol than wild-type mice (23). The targeted disruption of specific NPY receptors alters ethanol consumption, and selective NPY receptor antagonists suppress ethanol intake in P C57BL/6J mice (24). NPY expression levels, similar to phosphorylated CREB levels, have been reported to be lower in the $\mathrm{CeA}$ of $\mathrm{P}$ rats compared to NP rats (25).
In this issue of the JCI, Pandey and coworkers show that $\mathrm{P}$ rats displayed higher baseline anxiety-like behaviors and consumed greater amounts of alcohol compared with NP rats (13), supporting prior observations (26). This finding prompted the investigators to posit that $\mathrm{P}$ rats drink excessive amounts of ethanol in order to reduce anxiety levels. Both investigatorand self-administered ethanol stimulated cAMP/PKA signaling in the CeA and medial amygdala (but not the basolateral amygdala), as evidenced by increased expression levels of the $\alpha$-catalytic subunit of PKA (PKA-C $\alpha$ ), phosphorylated CREB, and NPY. These changes were accompanied by a reduction in anxiety-like behavior in the $P$ rats (Figure 1). Impressively, the neurochemical and behavioral effects of alcohol were then mimicked by infusion of the PKA activator Sp-cAMP, or of NPY, into the CeA of these rats. In contrast, these manipulations did not produce any changes in anxiety levels or in phosphorylated CREBinduced NPY expression in the amygdaloid structures of NP rats. However, infusion of the PKA inhibitor Rp-cAMP into the
CeA provoked anxiety-like behaviors and increased alcohol intake in NP rats.

A provocative implication of these findings (13) is that decreased CREB function in the CeA may be operative in maintaining high anxiety levels and alcohol-drinking behaviors of $\mathrm{P}$ rats. These data need to be replicated and then placed in context with 2 other very important neurotransmitter systems in the amygdala: corticotropin-releasing factormediated (CRF-mediated) and GABA-mediated signaling. CRF is an anxiogenic neuropeptide. Microdialysis studies have measured increased extracellular levels of CRF in the CeA during acute ethanol withdrawal in rats (27). CRF antagonists have been shown to reverse the excessive drinking of ethanol associated with ethanol withdrawal (28). Likewise, behavioral and neurochemical studies have implicated GABAergic transmission in the CeA in regulating alcohol intake (29). Chronic administration of ethanol is associated with increased GABA release in the CeA, which is mediated in part through CRF (11).

Although the findings reported by Pandey and coworkers (13) are important, cautious interpretation is appropriate. While animal models employed to study alcohol-use disorders have been extremely helpful, they have limitations. Alcoholism is a complex behavioral disorder, the product of environmental and polygenic interactions. Equating the human experience of anxiety with a rodent's "choice" in an open field test may be stretching the limits of extrapolation. Furthermore, while rodent studies have demonstrated powerful efficacy for several classes of medications in reducing alcohol consumption, the effect of these drugs in treatment of alcohol-use disorders in humans has been, at best, weak (30). There is a clear need to develop more reliably predictive animal models for alcoholism.

With these caveats, the findings of Pandey and coworkers (13) are noteworthy. Their data inform us that we cannot limit our investigational focus to the mesolimbic DA system; the amygdala must be included when formulating models for alcohol abuse and dependence. These observations should prompt the pursuit of pharmacological manipulations of CREB and/or NPY expression in order to modify heavy alcohol intake.

Address correspondence to: Gary S. Wand, Johns Hopkins University School of Medicine, Ross Research Building, Room 863, 720 Rutland Avenue, Baltimore, Maryland 21205, USA. Phone: (410) 955-7225; Fax: (410) 955-0841; E-mail: gwand@jhmi.edu. 
1. Saitz, R. 2005. Clinical practice. Unhealthy alcohol use. N. Engl. J. Med. 352:596-607.

2. Grant, B.F. 1994. Alcohol consumption, alcohol abuse and alcohol dependence. The United States as an example. Addiction. 89:1357-1365.

3. Harwood, H.J., Fountain, D., and Livermore, G. 1998. Economic costs of alcohol abuse and alcoholism. Recent Dev. Alcohol. 14:307-330.

4. Rose, R. 1998. A developmental behavior-genetic perspective on alcoholism risk. Alcohol Health Res. World. 22:131-143.

5. Tupala, E., and Tiihonen, J. 2004. Dopamine and alcoholism: neurobiological basis of ethanol abuse [review]. Prog. Neuropsychopharmacol. Biol. Psychiatry. 28:1221-1247.

6. Oswald, L., McCaul, M., Wong, D., and Wand, G. 2005. Association of ventral striatal dopamine release with cortisol and drug liking. Neuropsychopharmacology. 30:821-832.

7. Yoder, K., et al. 2005. Dopamine D(2) receptor availability is associated with subjective responses to alcohol. Alcohol. Clin. Exp. Res. 29:965-970.

8. Volkow, N., Fowler, J., and Wang, G. 2004. The addicted human brain viewed in the light of imaging studies: brain circuits and treatment strategies. Neuropharmacology. 47(Suppl. 1):3-13

9. Liu, X., and Weiss, F. 2002. Reversal of ethanolseeking behavior by D1 and D2 antagonists in an animal model of relapse: differences in antagonist potency in previously ethanol-dependent versus nondependent rats. J. Pharmacol. Exp. Ther. 300:882-889.

10. Merikangas, K.R., et al. 1998. Comorbidity of substance use disorders with mood and anxiety disorders: results of the International Consortium in Psychiatric Epidemiology. Addict. Behav. 23:893-907.

11. Koob, G.F. 2003. Alcoholism: allostasis and beyond. Alcohol. Clin. Exp. Res. 27:232-243.
12. Willinger, U., et al. 2002. Anxiety as a predictor of relapse in detoxified alcohol-dependent patients. Alcohol Alcohol. 37:609-612.

13. Pandey, S.C., Zhang, H., Roy, A., and Xu, T. 2005. Deficits in amygdaloid cAMP-responsive elementbinding protein signaling may play a role in genetic predisposition to anxiety and alcoholism. J. Clin. Invest. 115:2762-2773. doi:10.1172/JCI24381.

14. Koob, G. 2004. A role for GABA mechanisms in the motivational effects of alcohol. Biochem. Pharmacol. 68:1515-1525

15. Li, T.-K., Lumeng, L., and Doolittle, D.P. 1993. Selective breeding for alcohol preference and associated responses. Behav. Genet. 23:163-170.

16. McClung, C.A., and Nestler, E.J. 2003. Regulation of gene expression and cocaine by CREB and $\Delta$ FosB. Nat. Neurosci. 6:1208-1215.

17. Hoffman, P., and Tabakoff, B. 1990. Ethanol and guanine nucleotide binding proteins: a selective interaction. FASEB J. 4:2612-2622.

18. Yang, X., Horn, K., Baraban, J.M., and Wand, G.S. 1998. Chronic ethanol administration phosphorylation of cyclic AMP-response element binding protein in granule cells of rat cerebellum. J. Neurochem. 70:224-232.

19. Constantinescu, A., Diamond, I., and Gordon, A.S 1999. Ethanol-induced translocation of cAMPdependent protein kinase to the nucleus. Mechanism and functional consequences. J. Biol. Chem. 274:26985-26991.

20. Li, J., Li, Y.-H., and Yuan, X.-R. 2003. Changes of phosphorylation of cAMP response binding protein in rat nucleus accumbens after chronic ethanol intake: naloxone reversal. Acta Pharmacol. Sin. 24:930-936.

21. Pandey, S.C., Roy, A., Zhang, H., and Xu, T. 2004 Partial deletion of the CREB gene promotes alcohol-drinking behaviors. J. Neurosci. 24:5022-5030.
22. Valdez, G., and Koob, G. 2004. Allostasis and dysregulation of corticotropin-releasing factor and neuropeptide Y systems: implications for the development of alcoholism. Pharmacol. Biochem. Behav. 79:67-689.

23. Thiele, T.E., Marsh, D.J., Ste. Marie, L., Bersntein, I.L., and Palmiter, R.D. 1998. Ethanol consumption and resistance are inversely related to neuropeptide Y levels. Nature. 396:366-369.

24. Thiele, T., and Badia-Elder, N. 2003. A role for neuropeptide $\mathrm{Y}$ in alcohol intake control: evidence from human and animal research. Physiol. Behav. 79:9-101

25. Suzuki, R., Lumeng, L., McBride, W.J., Li, T.-K., and Hwang, B.H. 2004. Reduced neuropeptide Y mRNA expression in the central nucleus of amygdala of alcohol preference and anxiety. Brain Res. 1014:251-254.

26. Kampov-Polevoy, A.B., Matthews, D.B., Gause, L., Morrow, A.L., and Overstreet, D.H. 2000. P rats develop physical dependence on alcohol via voluntary drinking: changes in seizure thresholds, anxiety, and patterns of alcohol drinking. Alcohol. Clin. Exp. Res. 24:278-284.

27. Merlo-Pich, E., and Weiss, F. 1998. Neurocircuitry targets in ethanol reward and dependence. Alcohol. Clin. Exp. Res. 22:3-9.

28. Valdez, G.R., Zorrilla, E.P., Roberts, A.J., and Koob, G.F. 2003. Antagonism of corticotropin-releasing factor attenuates the enhanced responsiveness to stress observed during protracted ethanol abstinence. Alcohol. 29:55-60

29. Hyytia, P., and Koob, G. 1995. GABAA receptor antagonism in the extended amygdala decreases ethanol self-administration in rats. Eur. J. Pharmacol. 283:151-159.

30. Krystal, J.H., et al. Naltrexone in the treatment of alcohol dependence. N. Engl. J. Med. 345:1734-1739.

\title{
Shifting gears: liver SR-BI drives reverse cholesterol transport in macrophages
}

\author{
Astrid E. van der Velde and Albert K. Groen \\ Academic Medical Center Liver Center, Academic Medical Center, Amsterdam, The Netherlands.
}

\begin{abstract}
Cholesterol efflux from macrophages, the first step in reverse cholesterol transport (RCT), is assumed to play a critical role in the pathogenesis of atherosclerosis. However, in vivo proof supporting this hypothesis is lacking, due to difficulties in determining the activity of this first step in RCT. In this issue of the JCI, Zhang et al. apply their recently developed method for measuring RCT in vivo to estimate RCT in mouse models with varying levels of HDL turnover. A surprisingly efficient clearance of cholesterol to feces is observed in mice overexpressing hepatic scavenger receptor class B type I (SR-BI), whereas in $S R-B I-$ knockout mice, cholesterol clearance is diminished (see the related article beginning on page 2870). The study demonstrates that hepatic SR-BI is a positive regulator of macrophage RCT in vivo.
\end{abstract}

Nonstandard abbreviations used: RCT, reverse cholesterol transport; SR-BI, scavenger receptor class B type I.

Conflict of interest: The authors have declared that no conflict of interest exists.

Citation for this article: J. Clin. Invest. 115:2699-2701 (2005). doi:10.1172/JCI26241.
The transport of excess cholesterol from the periphery into the liver and bile, followed by excretion in the feces, is defined (Figure 1). Since the original definition of RCT by Glomset and Norum in 1973 (1), this pathway has become increasingly as reverse cholesterol transport (RCT) popular as a target for therapeutic strategies aimed at achieving the regression of atherosclerosis. Theoretically, a lipid-laden macrophage can release its contents by activation of efflux pathways. HDL is considered to be the primary cholesterol carrier in RCT, but conclusive evidence for this contention has been lacking until now. It is not an easy task to estimate net cholesterol flux from peripheral tissues to feces. Neutral sterols found in feces are derived from several sources. The major source is the liver, where the bulk of body cholesterol and bile salts are synthesized. After their secretion from the liver in bile, both components are secreted into the intestine, where up to $95 \%$ of bile salts and $30-60 \%$ of cholesterol is reabsorbed. The nonabsorbed cholesterol partly undergoes bacte- 